\title{
Hipertensão ocular recorrente durante hemodiálise em paciente portador de glaucoma
}

\author{
Recurrent intraocular hypertension during hemodialysis in glaucoma patient

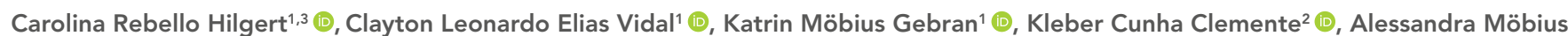 \\ Gebran $^{1}$ (1), Gabriel Rebello Hilgert ${ }^{1,3}$ (1)

\section{Descritores:}

Glaucoma neovascular; Diálise renal; Pressão intraocular, Humor aquoso; Concentração osmolar

Keywords:

Glaucoma, neovascular; Renal dialysis; Intraocular pressure; Aqueous humor; Osmolar concentration

Recebido: 03/11/2019

Aceito:

21/8/2021

Autor correspondente: Carolina Rebello Hilgert Avenida Rubens Gil de Camillo, 83 Chácara Cachoeira CEP: 79040-090 - Campo Grande, MS,$$
\text { Brasil }
$$

E-mail: carolrhilgert@gmail.com

Instituição de realização do trabalho: instituto da Visão de Mato Grosso do Sul Campo Grande, MS, Brasil.

Fonte de auxílio à pesquisa: trabalho não financiado.

Conflitos de interesse: não há conflitos de interesses.

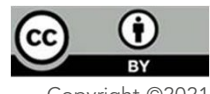

Copyright (C2021

\section{RESUMO}

A hipertensão ocular aguda durante a hemodiálise constitui evento raro e pode ser causa relevante de interrupção do tratamento dialítico devido à dor. Relata-se o caso de um paciente de 70 anos de idade, do sexo masculino, que apresentou quadros recorrentes de intensa dor ocular unilateral durante sessões dialíticas devido ao aumento de pressão intraocular. $\bigcirc$ paciente era portador de grave diminuição da acuidade visual no olho direito devido a glaucoma neovascular, controlado com medicação hipotensora tópica. Uma hora após o início da sessão dialítica, apresentou dor excruciante no olho direito, sendo necessário interromper o tratamento por diversas vezes. A dor somente era amenizada com uso de opioides por via endovenosa ou após cerca de 6 horas do procedimento. Injeção intraocular de drogas antiangiogênicas e acetazolamida por via oral, assim como tratamentos tradicionais para quadros agudos de hipertensão intraocular, como uso de hipotensor tópico e medicamentos hiperosmolares, foram insuficientes para o controle da dor. $\bigcirc$ problema se resolveu com ciclofotocoagulação transescleral realizada com laser diodo, com redução da pressão intraocular basal e controle da dor, o que permitiu a realização de sessões completas de hemodiálise. A base fisiopatológica desse evento incomum e suas opções terapêuticas são discutidas aqui.

\section{ABSTRACT}

Acute ocular hypertension during hemodialysis is a rare event and may lead to interruption of dialytic therapy due to pain. A case of a 70-year-old male patient is reported, who presented recurrent intense unilateral ocular pain episodes during dialysis sessions for increased intraocular pressure. The patient presented with severely decreased visual acuity in the right eye due to neovascular glaucoma, which was controlled with topical hypotensive medication. One hour after initiating dialysis, he presented an excruciating pain on the right eye, which required interruption of treatment several times. Pain relief was possible only with intravenous opioids, or approximately 6 hours after dialysis. Intraocular injection of antiangiogenic drugs and per oris acetazolamide, as well as other traditional treatments for acute episodes of intraocular hypertension, such as topical antihypertensive agents and hyperosmotic medications, were not sufficient to control pain. The problem was solved with transscleral diode laser cyclophotocoagulation, which reduced baseline intraocular pressure and controlled pain, enabling complete hemodialysis sessions. The pathophysiological aspects and therapeutic options of this unusual condition are discussed 


\section{INTRODUÇÃO}

A hipertensão ocular durante a hemodiálise (HD) é um evento ainda envolto em controvérsias. Enquanto é possível observar sua ocorrência em pacientes portadores de glaucoma ou não, ${ }^{(1-3)}$ também há relatos da redução da pressão intraocular (PIO) durante a sessão dialítica. ${ }^{(4)}$

Entre as principais complicações associadas à HD, encontram-se hipotensão arterial, câimbras musculares, cefaleia, distúrbios eletrolíticos, náuseas e vômitos. Mais raramente, arritmias cardíacas, aumento da PIO e dispneia são relatados. ${ }^{(5)}$ As complicações oculares podem ocorrer durante ou após a HD. A elevação da PIO comumente ocorre em portadores de glaucoma, associada ou não à dor ocular ou à visão turva. ${ }^{(6)}$

A alteração da PIO durante sessões de HD foi descrita pela primeira vez por Sitprija et al., em 1964. ${ }^{(7)}$ Os efeitos da HD em parâmetros oculares variam muito, estando a fisiopatologia do problema ainda não definitivamente elucidada. ${ }^{(4,7)}$ Tal fato, somado a alterações anatômicas ou funcionais do globo ocular, que, porventura, sobrecarreguem o sistema de drenagem do humor aquoso (HA) pode predispor à ocorrência de hipertensão ocular e de suas complicações. ${ }^{(8)}$

Esse pode ser o caso de pacientes diabéticos, que formam um importante contingente de indivíduos que se submetem rotineiramente à HD. Esses pacientes, em especial os portadores de retinopatia diabética, são mais propensos a desenvolver neovascularização do seio camerular, hipertensão ocular e glaucoma neovascular.(9) Alterações da PIO durante a HD nessa população fazem deste um tema de interesse, o qual é esparsamente descrito na literatura. ${ }^{(6,9)}$

Assim, objetivou-se relatar um caso de dor ocular intensa secundária à hipertensão ocular grave e recorrente durante a realização de HD, em portador de glaucoma neovascular. Os prováveis eventos fisiopatológicos envolvidos e opções terapêuticas para seu manejo são avaliados.

\section{RELATO DE CASO}

Paciente do sexo masculino, 70 anos, coronariopata, portador de glaucoma neovascular no olho direito (OD) e insuficiência renal crônica decorrente de nefroesclerose secundária à hipertensão arterial sistêmica descontrolada, apresentava episódios recorrentes de dor excruciante no OD durante a HD.

O paciente já tinha utilizado acetazolamida por via oral (VO) antes das sessões dialíticas, mas não houve alívio nos episódios dolorosos. A dor somente melhorava com o uso de morfina por via endovenosa (EV) ou interrupção da HD.

O paciente fora submetido à cirurgia de catarata sem intercorrências em ambos os olhos há 2 anos. Apresentou perda súbita de visão em OD 8 meses antes por obstrução de veia central da retina, a qual foi tratada com fotocoagulação com laser de argônio e aplicação intravítrea de bevacizumabe, sem melhora da acuidade visual e com evolução para perda de percepção luminosa.

À biomicroscopia, apresentava OD com córnea transparente, midríase média paralítica, discoria e ectrópio uveal com presença de rubeosis iridis (Figura 1). O olho esquerdo (OE) não apresentava alterações. A gonioscopia de OD mostrava extensas áreas de goniossinéquias, com presença de neovasos. O OE apresentava ângulo do seio camerular aberto, com estruturas anatômicas visíveis e bem definidas, sem a presença de neovasos.

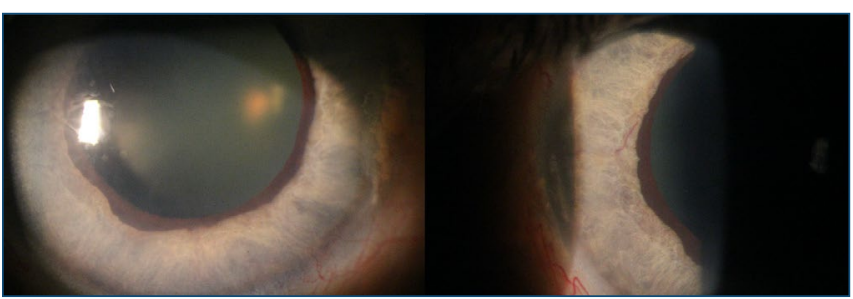

Figura 1. Olho direito calmo, córnea transparente, midríase média paralítica, discoria e ectrópio uveal com presença de rubeosis iridis.

O glaucoma neovascular no OD era controlado com terapia tríplice hipotensora tópica, feita com travoprosta (Sanofi Medley Farmacêutica LTDA, Suzano, SP, Brasil), tartarato de brimonidina (Novartis Biociências S.A., Anápolis, GO, Brasil) e cloridrato de dorzolamida (Biosintética Farmacêutica LTDA, São Paulo, SP, Brasil), e a PIO basal era de $15 \mathrm{mmHg}$ no OD e $12 \mathrm{mmHg}$. Não fazia uso de medicações em $\mathrm{OE}$.

Como o paciente relatava dor excruciante durante a sessão de HD, a PIO do OD foi avaliada com Tono-Pen Avia ${ }^{\circledR}$ (Reichert Inc., Nova Iorque, Estados Unidos), evidenciando-se aumento progressivo da PIO durante a HD em ambos os olhos, mas mais intensamente em OD. Esse aumento foi também observado mesmo quando o paciente estava assintomático (Figura 2). A dor intensa e aguda, em geral, somente ocorria no início do processo dialítico, no OD, quando a PIO aferida era de $42 \mathrm{mmHg}$ (Figura 3). A dor incapacitante não permitia finalizar a sessão dialítica. 


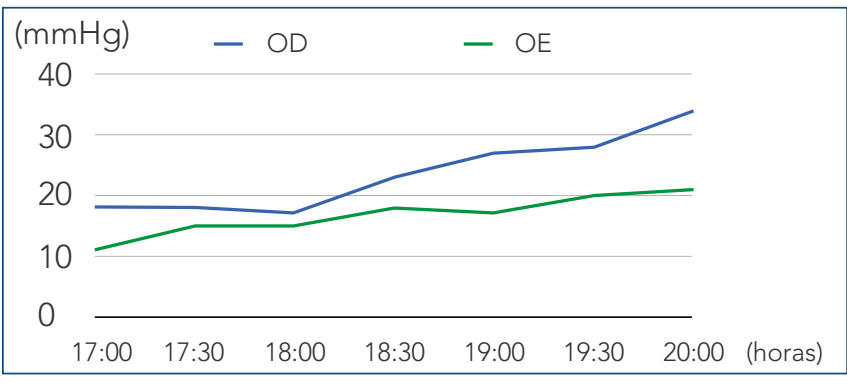

Figura 2. Comportamento da pressão intraocular durante hemodiálise na primeira avaliação aferida por Tono-Pen Avia®.

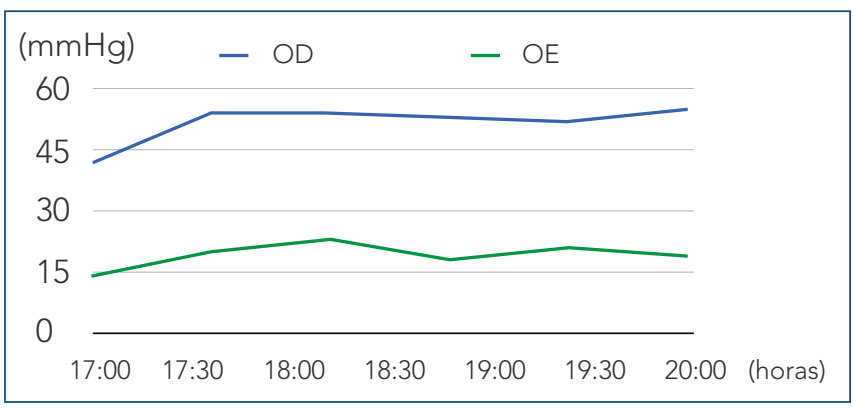

OD: olho direito; OE: olho esquerdo.

Figura 3. Comportamento da pressão intraocular durante hemodiálise na segunda avaliação aferida por Tono-Pen Avia®.

Por se tratar de um olho amaurótico e doloroso, foi realizada ciclofotocoagulação transescleral com laser diodo (Iridex, Buffalo, Nova Iorque, Estados Unidos), resultando em normalização da PIO sem medicações (12mmHg) e controle da dor.

Após 4 meses, no entanto, observou-se novo aumento da PIO basal (22mmHg), e, mesmo com a reintrodução de medicação hipotensora tópica, houve reincidência dos episódios álgicos durante a HD. Foi, então, realizada nova sessão de ciclofotocoagulação transescleral, com posterior diminuição da PIO e ausência de eventos dolorosos.

A avaliação 14 meses após a última sessão de ciclofotocoagulação mostrou o paciente assintomático, com PIO controlada ( $8 \mathrm{mmHg}$ ) sem medicação hipotensora e capaz de concluir suas sessões de HD sem intercorrências oculares.

Foi indicada monitorização cuidadosa de seu olho contralateral, devido à possibilidade de flutuações da PIO durante as sessões dialíticas.

\section{DISCUSSÃO}

Os autores descrevem um evento incomum de aumento abrupto da PIO durante sessões de HD em olho portador de glaucoma neovascular.

No processo dialítico, solutos são removidos do plasma, levando a uma redução de sua osmolaridade. Por conseguinte, a maior pressão osmótica exercida no fluido intraocular leva a um maior aporte de água para o HA, culminando na elevação da PIO. ${ }^{\left({ }^{10)}\right.}$ Apesar de não completamente esclarecida, essa é atualmente a principal hipótese causal para alterações da PIO durante HD.

O termo "desequilíbrio dialítico ocular" (ocular dialysis disequilibrium) foi proposto para caracterizar esse fenômeno. ${ }^{(2)}$ Além do aumento da PIO, observou-se que pode ocorrer diminuição da pressão de perfusão. ${ }^{(11)}$

Em síntese, a PIO é a resultante de três fatores: produção de HA, facilidade de escoamento desse líquido pelo trabeculado e pressão venosa episcleral..$^{(12)}$ A variabilidade anatômica na população que se submete à HD também deve ser considerada, visto que uma dificuldade preexistente de escoamento do há, associada ao aporte adicional pela HD, pode elevar ainda mais a PIO, como neste caso.

Há variações nas descrições de PIO durante HD, com relatos de acréscimo, decréscimo ou equilíbrio da PIO. ${ }^{(13)}$ Nos casos de decréscimo da PIO, argumenta-se que a variação rápida no gradiente de osmolaridade não seria suficiente para provocar alterações bruscas da PIO, postulando-se que, em situações em que o sistema de escoamento encontra-se funcionalmente saudável, este se adaptaria ao maior aporte de HA. ${ }^{(8,14-16)}$

A diminuição da profundidade da câmara anterior durante a HD, aferida tanto por biometria, quanto por tomografia de coerência óptica (OCT, sigla do inglês optical coherence tomography), pode ser considerada fator adicional a romper esse equilíbrio em olhos predispostos a fechamento angular. ${ }^{(3,15,17)}$ Portadores de glaucoma de ângulo fechado, ângulo oclusível ou neovascularização angular seriam, então, mais suscetíveis ao desequilíbrio nesse sistema de drenagem, com consequente resposta hipertensiva ocular. ${ }^{(18)}$

O uso de soluções hiperosmóticas mostrou alta efetividade no controle da PIO e baixa taxa de complicações em pacientes portadores de glaucoma..$^{(1)}$ Entretanto, o paciente deste caso, por razões sistêmicas, tinha contraindicado o uso de soluções hiperosmóticas.

A acetazolamida por VO é uma potencial ferramenta para o controle da PIO durante a HD. ${ }^{(9)}$ Entretanto, a utilização de inibidores da anidrase carbônica é contraindicada em dialíticos, pelo risco de precipitar acidose metabólica. ${ }^{(6)}$

Para o presente caso de um portador de olho amaurótico doloroso com dificuldade em alterar o protocolo dialítico no longo prazo, foi realizada ciclofotocoagulação transescleral. Essa foi uma alternativa encontrada para evitar a alteração da conduta sistêmica e do protocolo dialítico em paciente idoso, portador de múltiplas comorbidades. 
Com isso, opções cirúrgicas mais invasivas puderam ser evitadas, visto que a ciclofotocoagulação transescleral com laser diodo se mostrou eficaz no controle da hipertensão ocular e dor. ${ }^{(19)}$

Modificações na prescrição dialítica que levem ao aumento na tonicidade plasmática diminuem o desequilíbrio entre o plasma e os líquidos intraoculares, podendo prevenir o aumento da PIO. ${ }^{(1,2,20)}$ No caso em questão, a glicose hipertônica endovenosa não chegou a ser administrada. Contudo, ela poderia ser uma alternativa a ser considerada durante a HD em situações como a do paciente deste relato, principalmente em se tratando de indivíduos não diabéticos. ${ }^{(6)}$

\section{CONCLUSÃO}

A hipertensão ocular aguda e recorrente pode inviabilizar a realização da hemodiálise em portadores de glaucoma neovascular. O tratamento clínico convencional da hipertensão ocular não é habitualmente efetivo para controlar sintomas álgicos nessas situações. Tais quadros devem ser abordados em conjunto com o clínico ou o nefrologista assistente, a fim de definir a melhor opção terapêutica, que pode incluir alterações no protocolo dialítico, injeções de glicose hipertônica ou, ainda, alternativas cirúrgicas. A realização de ciclofotocoagulação transescleral pode ser efetiva em olhos portadores de baixo prognóstico visual.

\section{REFERÊNCIAS}

1. Tokuyama $T$, Ikeda $T$, Sato K. Effect of plasma colloid osmotic pressure on intraocular pressure during haemodialysis. $\mathrm{Br} J$ Ophthalmol. 1998;82(7):751-3.

2. Lippold CL, Kalarn SP, Swamy RN, Patel AM. Ocular dialysis disequilibriumManagement of intraocular pressure during hemodialysis of open angle glaucoma: A case report and review of the literature. Hemodial Int. 2019;23(3):E72-7.

3. Gracitelli CP, Stefanini FR, Penha F, Góes MÂ, Draibe SA, Canziani ME, et al. Anterior chamber depth during hemodialysis. Clin Ophthalmol. 2013;7:1635-9.
4. Liakopoulos V, Demirtzi P, Mikropoulos DG, Leivaditis K, Dounousi E, Konstas AG. Intraocular pressure changes during hemodialysis. Int Urol Nephrol. 2015;47(10):1685-90. [erratum in Int Urol Nephrol. 2015;47(10):1691].

5. de Souza Terra F, Maria A, Dias Costa D, Tavares de Figueiredo E, Moterani de Mo-Rais A, Dias Costa M, et al. As principais complicações apresentadas pelos pacientes renais crônicos durante as sessões de hemodiálise. Rev Bras Clínica Médica. 2010;8(3):187-92.

6. Frezzotti P, Menicacci C, Bagaglia SA, Mittica P, Toto F, Motolese I. Management of intraocular pressure elevation during hemodialysis of neovascular glaucoma: a case report. BMC Ophthalmol. 2016;16(1):23.

7. Sitprija V, Holmes JH, Ellis PP. Changes in intraocular pressure during hemodialysis. Invest Ophthalmol. 1964;3:273-84.

8. Burn RA. Intraocular pressure during haemodialysis. $\mathrm{Br} \mathrm{J}$ Ophthalmol. 1973;57(7):511-3.

9. Song WK, Ha SJ, Yeom HY, Seoung GJ, Hong YJ. Recurrent intraocular pressure elevation during hemodialysis in a patient with neovascular glaucoma. Korean J Ophthalmol. 2006;20(2):109-12.

10. Goel M, Picciani RG, Lee RK, Bhattacharya SK. Aqueous humor dynamics: a review. Open Ophthalmol J. 2010;4(1):52-9.

11. Hu J, Bui KM, Patel KH, Kim H, Arruda JA, Wilensky JT, et al. Effect of hemodialysis on intraocular pressure and ocular perfusion pressure. JAMA Ophthalmol. 2013;131(12):1525-31.

12. Weinreb RN, Aung T, Medeiros FA. The pathophysiology and treatment of glaucoma: a review. JAMA. 2014;311(18):1901-11

13. Cecchin E, De Marchi S, Tesio F. Intraocular pressure and hemodialysis. Nephron. 1986;43(1):73-4

14. Hojs R, Pahor D. Intraocular pressure in chronic renal failure patients treated with maintenance hemodialysis. Ophthalmologica. 1997;211(6):325-6.

15. Barbosa CP, Stefanini FR, Penha F, Góes MA, Draibe SA, Canziani ME, et al. Intraocular pressure and ocular perfusion during hemodialysis. Arq Bras Oftalmol. 2011;74(2):106-9

16. Chelala E, Dirani A, Fadlallah A, Slim E, Abdelmassih Y, Fakhoury H, et al. Effect of hemodialysis on visual acuity, intraocular pressure, and macular thickness in patients with chronic kidney disease. Clin Ophthalmol. 2015;9:109-14.

17. Shin YU, Kim JH, Cho H, Kim DS, Yi JH, Han SW, et al. Effect of hemodialysis on anterior chamber angle measured by anterior segment optical coherence tomography. J Ophthalmol. 2019;2019:2406547.

18. Fischer MD, Fleischhauer J, Keusch G, Abegg MH. Rise in intraocular pressure during haemodialysis in a patient with reduced outflow facility. $\mathrm{Br}$ J Ophthalmol. 2007;91(8):1091-3.

19. Nabili S, Kirkness CM. Trans-scleral diode laser cyclophoto-coagulation in the treatment of diabetic neovascular glaucoma. Eye (Lond). 2004;18(4):352-6.

20. Leiba H, Oliver M, Shimshoni M, Bar-Khayim Y. Intraocular pressure fluctuations during regular hemodialysis and ultrafiltration. Acta Ophthalmol (Copenh). 1990;68(3):320-2. 